\title{
¡TODO BIEN! EL RETO DE MEJORAR LAS RELACIONES INTERPERSONALES EN ADOLESCENTES ESCOLARIZADOS COLOMBIANOS
}

\author{
¡ALL GOOD! THE CHALLENGE \\ OF IMPROVING INTERPERSONAL \\ RELATIONSHIPS IN COLOMBIAN \\ SCHOOLING ADOLESCENTS
}

\section{Mauricio Herrera-López ${ }^{1}$}

\section{Eva Romera²}

\section{Rosario Ortega-Ruiz ${ }^{3}$}

Universidad de Naríño-Universidad de Córdoba, España

\section{RESUMEN}

Las relaciones interpersonales entre adolescentes son relevantes en el aprendizaje y la convivencia escolar. Se analizó las dimensiones individuales y contextuales que influyen en las relaciones interpersonales entre adolescentes. Participaron 1084 adolescentes escolarizados

1 Profesor Asociado, Departamento de Psicología, Universidad de Nariño (Colombia). Doctor en Ciencias Sociales y Jurídicas, Investigador del grupo Psicología y Salud (Udenar) y del LAECOVI "Laboratorio de Estudios sobre Convivencia y Prevención de la Violencia" de la Universidad de Córdoba (España). Correo electrónico: mherrera@udenar.edu.co. ORCID: https:// orcid.org/0000-0002-0292-2688

2 Profesora Titular, Departamento de Psicología, Universidad de Córdoba (España). Doctora en Psicología Aplicada. Directora del Máster en Psicología Aplicada a la Educación y el Bienestar Social. Investigadora del LAECOVI "Laboratorio de Estudios sobre Convivencia y Prevención de la Violencia" de la Universidad de Córdoba (España). Correo electrónico: eva.romera@ uco.es. ORCID: https://orcid.org/0000-0002-9414-8019

3 Profesora Emérita, Departamento de Psicología, Universidad de Córdoba (España). Doctora en Psicología. Directora del LAECOVI "Laboratorio de Estudios sobre Convivencia y Prevención de la Violencia" de la Universidad de Córdoba (España). Editora Asociada de la revista Educational Psychology. Co-editora de la revista Psychology, Society and Education. Correo electrónico: ortegaruiz@uco.es. ORCID: https://orcid.org/0000-0003-2110-6931 
(52.8\% mujeres) del sur de Colombia, con edades entre 11 y 19 años $(M=14.9 ; S D=1.89)$. Se analizaron las propiedades psicométricas de las escalas utilizadas. Un modelo de ecuaciones estructurales informó las relaciones e influencias de la empatía, prosocialidad, metas sociales, autoeficacia social y ajuste normativo sobre la calidad de sus relaciones interpersonales (ajuste social). Se encontraron óptimas propiedades psicométricas para las escalas y adecuados ajustes del modelo explicativo-predictivo; éste indicó que la empatía, prosocialidad y autoeficacia social con los compañeros predicen la calidad de las relaciones interpersonales. Resalta la influencia negativa de la autoeficacia social con profesores hacia la calidad de las relaciones, y del ajuste normativo sobre el desempeño social con compañeros. Se discuten orientaciones para el desarrollo de la prosocialidad y empatía.

\section{PALABRAS CLAVE}

Relaciones interpersonales, empatía, metas sociales, autoeficacia social, ajuste social y normativo.

\section{ABSTRACT}

Interpersonal relationships between adolescents are relevant in learning and school coexistence. The individual and contextual dimensions that influence interpersonal relationships among adolescents were analyzed. Participants were 1084 adolescents (52.8\% women) from Colombia, aged between 11 and 19 years $(M=$ $14.9, S D=1.89$ ). The psychometric properties of the scales used were analyzed. A model of structural equations informed the relationships and influences of empathy, prosociality, social goals, social self-efficacy and normative adjustment on social adjustment. Optimal psychometric properties were found for the scales and adequate adjustments of the explanatory model; this indicated that empathy, prosociality and social self-efficacy with peers predict the quality of interpersonal relationships. It highlights the negative influence of social selfefficacy with teachers towards the quality of relationships, and of the normative adjustment on social performance with peers. Guidelines for the development of prosociality and empathy are discussed.

\section{KEYWORDS}

Interpersonal relationships, empathy, social goals, social self-efficacy, social and normative adjustment

La sociología y la psicología social han definido las relaciones interpersonales como las interacciones que acontecen en el marco de estructuras y prácticas bien definidas por sistemas sociales 0 institucionales preestablecidos como la escuela, la comunidad o la familia (Giddens, 1984). Se trata de procesos relacionales entre personas que comparten contextos, actividades e intereses comunes (Bandura, 2001). La psicología social aplicada a la educación (Maras, 2007) se ha interesado por estudiar el funcionamiento y las dimensiones implicadas en las relaciones interpersonales en el contexto de la escuela, en donde acontecen procesos dinámicos, como los que se derivan de las actividades de aprendizaje y enseñanza, que provocan el establecimiento y adopción de conductas, roles y en general, procesos complejos que siempre implican relaciones interpersonales (Bandura et al., 2003).

El estudio de las relaciones interpersonales tiene en los trabajos sobre relaciones entre iguales una referencia importante, ya que los sistemas sociales escolares siempre incluyen la comunicación y la actividad conjunta de grupos como los estudiantes (Ortega y MoraMerchán, 1996). Así, desde los años ochenta se han acrecentado y diversificado estos estudios sobre las relaciones entre iguales (Day et al., 2014; Hartup, 2005), relaciones que, además de promover la práctica y mejoramiento de 
los aprendizajes sociales, permiten que se generalicen o trasciendan de un ámbito a otro y se retroalimenten dependiendo de los éxitos o fracasos obtenidos (Hamm y Faircloth, 2005). En la actualidad es un tema de interés en parte debido al auge vertiginoso de los nuevos espacios de interacción como el ciberespacio, que ha reorientado el estudio de las prácticas de las relaciones interactivas en el marco de las redes sociales, tanto directas como virtuales (Casas et al., 2013).

Los estudios sobre las relaciones entre iguales en el campo de la psicología evolutiva han destacado la importancia de éstas como contexto de desarrollo (Hartup, 2005). Desde los estudios de Piaget (1936) sobre formación del criterio moral, la psicología del desarrollo reconoce que es en el contexto de iguales donde tienen lugar procesos interactivos que estimulan el aprendizaje de pautas de conducta de reciprocidad social y moral. Posteriormente, Hartup (2005) extendió el valor del contexto de iguales como un ámbito de desarrollo y aprendizaje a incluir en el conjunto de los más relevantes (familia y escuela), indicando que ciertos dominios y competencias difícilmente se lograrían sin los complejos procesos de relaciones interpersonales en los que tienen lugar las actividades y procesos que los entrenan.

A partir de los años de la escolaridad primaria, los procesos interactivos entre iguales van aumentando su relevancia y llegan a ser determinante en los años de la adolescencia, pues en esta edad las relaciones entre iguales favorecen el aprendizaje de múltiples habilidades y competencias sociales, así como de las normas y reglas morales (Del Rey et al., 2008; La Greca y López, 1998).

En el proceso evolutivo de socialización, la familia, la comunidad local y la escuela son relevantes ámbitos sociales en donde los adolescentes establecen relaciones interpersonales; en la familia se establecen fundamentalmente con los progenitores, pero también con los hermanos y otros miembros; en la comunidad local se establecen con los amigos y otros adultos, mientras que, en la escuela en todos los periodos del desarrollo, resultan relevantes las relaciones interpersonales con los compañeros y con los profesores (Bronfenbrenner, 2005). Este último ámbito se considera un contexto multidimensional y desafiante, pues en él se desarrollan interacciones complejas y con multiplicidad de vínculos que ofrece a los escolares diversas oportunidades para la adquisición de habilidades y competencias sociales, el cumplimiento de las responsabilidades en el aula, la aceptación de la autoridad del profesor y la negociación interpersonal favorecida por las interacciones con el grupo de iguales (Day et al., 2014; Ortega y Mora-Merchan, 2008; Rodkin y Ryan, 2012).

La configuración y desarrollo de las relaciones interpersonales entre iguales está determinada por múltiples dimensiones o dominios. Una a resaltar es la empatía (Decety y Svetlova, 2012; Martinez et al., 2021). Ésta se define como la capacidaddeentender,comprender (componente cognitivo) e implicarse (componente afectivo) en el estado emocional de los demás (Carré et al., 2013; Jolliffe y Farrington, 2006). La empatía es considerada una dimensión mediadora del aprendizaje social, particularmente en contextos familiares y escolares (Fernández-Pinto et al, 2008; Van-Lissa et al., 2014).

Otra dimensión es la prosocialidad. Ésta ha sido reconocida como aquella conducta que, sin la búsqueda de recompensas externas, favorece a otra persona o grupos, aumentando la probabilidad de generar una reciprocidad positiva, cooperativa, de calidad y solidaria en las relaciones interpersonales consecuentes (Eisenberg et al., 2006; Roche, 1995). Algunos aspectos referidos a la prosocialidad son la ayuda física y verbal, la disposición para 
dar, servir y compartir, el consuelo verbal, la escucha profunda y la valoración positiva del otro (Roche, 1998). Varias investigaciones sugieren que aporta positivamente a la disposición para comprender las emociones de los demás y beneficia el desarrollo de relaciones interpersonales de calidad (Gilman y Anderman, 2006).

Las metas sociales también hacen parte de las dimensiones consideradas relevantes en el desarrollo de las relaciones interpersonales entre iguales. Éstas se refieren a las representaciones cognitivas sobre los resultados deseados en el ámbito social (Méndez-Giménez et al., 2012), las cuales se construyen a partir de los objetivos que las personas quieren lograr, por tanto, son rutas que orientan el comportamiento (Dupont et al., 2014; Rodkin et al., 2013). Según Ryan y Shim (2006) existen tres tipos de metas sociales en la adolescencia: las metas de desarrollo (promoción), que se relacionan con la generación de autoaprendizajes sociales y con los esfuerzos para el desarrollo y mejora de las habilidades interpersonales; las metas de aproximación, que aluden a la búsqueda de popularidad y la obtención de juicios positivos por parte de los demás para demostrar que la persona es socialmente deseable; y por último, las metas de evitación, que implican evitar hacer cosas que podrían generar un juicio negativo, indicando deficiencia social. Algunos estudios sugieren que la presencia de metas sociales favorece la construcción de responsabilidad social y la adherencia a las normas sociales (Patrick et al., 1997); en particular, las metas de promoción se asocian con el ajuste social, pues se relacionan con la presencia de conductas adaptativas, con bajos niveles de agresión y una elevada prosocialidad (Rodkin et al., 2013; Ryan y Shim, 2006).

Algunas investigaciones han demostrado también que la autopercepción de eficacia en el desempeño social y el sentirse aceptado en un grupo favorecen la actitud positiva y optimista frente a la resolución de los conflictos que eventualmente surgen en las interrelaciones entre los escolares, pues influye en los niveles de compromiso social, en la capacidad de análisis de problemas, la detección de obstáculos y reconocimiento de consecuencias (Connolly, 1989); por tanto aporta positivamente a la autoconfianza del sujeto y retroalimentan la práctica de los aprendizajes sociales (Brouzos et al., 2014).

El estudio de las relaciones interpersonales entre iguales también incluye algunas variables del contexto como el ajuste normativo. Éste hace alusión a las actitudes y comportamientos referidos al cumplimiento de las normas básicas que favorecen la convivencia e interrelación de los sujetos en distintos contextos (Córdoba et al., 2016). Algunas investigaciones sugieren que la aceptación o rechazo de un adolescente por parte de los iguales está relacionado con el cumplimiento de las normas, pues, al parecer se rechaza con mayor probabilidad a quien las asume de manera innegociable, en comparación a quien no lo hace o las asume de manera más flexible (García et al., 2010; Rodkin y Ryan, 2012).

Finalmente, un aspecto importante de las relaciones interpersonales es la calidad con las que éstas se establecen (Segrin y Taylor, 2007). Dicha calidad se refiere a los comportamientos y actitudes positivas que favorecen un mejor ajuste social, como la cooperación, la solidaridad, el interés y atención hacia los demás, la defensa de los derechos propios de manera asertiva y la integración en actividades de grupo (Poorthuis et al., 2013; Smithyman et al., 2014). Importantes estudios han demostrado que la presencia de relaciones interpersonales de calidad no solo beneficia el clima escolar y la resolución de conflictos, sino que también reduce el riesgo frente al bullying, cyberbullying (Casas et al., 2013), el consumo de sustancias, la ansiedad 
social, la depresión y el suicidio (Elledge et al., 2013; Hinduja y Patchin, 2013; McGloin et al., 2014).

Así pues, se reconoce un panorama investigativo que sugiere que en el establecimiento y logro de calidad en las relaciones interpersonales entre iguales, participan múltiples dimensiones pertenecientes a la psicología del sujeto $\mathrm{y}$ del contexto social (Buhrmester et al., 1988; Gutiérrez et al., 2011; Herrera et al., 2016), sin embargo, se reconoce la necesidad de realizar investigaciones que las estudien en conjunto, desde metodologías multivariantes, pues se reconocen estudios que las asumen por separado; este propósito además permitiría una mejor comprensión de las condiciones particulares en las cuales se desarrollan (Poorthuis et al., 2013).

A partir de lo argumentado anteriormente, se propuso como objetivo determinar las relaciones e influencias de la empatía, la prosocialidad, las metas sociales, la autopercepción de eficacia social y el ajuste normativo, sobre la calidad de las relaciones interpersonales entre iguales en la escuela. Para esto, inicialmente se reconocieron las propiedades psicométricas de todas las escalas utilizadas y adaptarlas al contexto.

Se partió de la hipótesis: la empatía, la prosocialidad, las metas sociales, la autopercepción de eficacia en el desempeño social y el ajuste normativo predecirán la calidad de las relaciones interpersonales entre iguales en la escuela.

\section{MÉTODO}

\section{PARTICIPANTES}

La muestra fue incidental y estuvo conformada por 1084 estudiantes colombianos (52.8\% mujeres y $47.2 \%$ hombres) adolescentes escolarizados en todos los cursos de Educación Media (Bachillerato); así, el $17.7 \%$ cursaban $6^{\circ}$ grado, el $18.3 \% 7^{\circ}$, el $20.1 \% 8^{\circ}$ grado, el $16 \% 9^{\circ}$, el
$12.5 \% 10^{\circ}$ y el $15.4 \% 11^{\circ}$ grado. Los estudiantes pertenecían a 8 colegios del sector urbano de San Juan de Pasto, Colombia, a saber: Liceo de la Universidad de Nariño (12\%), Instituto Champagnat (4.6\%), I.E.M. Ciudad de Pasto (15.9\%), INEM Luis Delfín Insuasty Rodríguez (11.4\%), I.E.M. San Juan Bosco (17.1\%), I.E.M. Heraldo Romero Sánchez (10.3\%), Colegio San Felipe Neri (14.8\%) y Colegio Filipense Nuestra Señora de la Esperanza (13.9\%). Las edades de los participantes estaban comprendidas entre los 11 y los 19 años $(M=14.9 ; S D=1.89)$.

\section{INSTRUMENTOS}

Para medir la empatía se utilizó una versión abreviada del cuestionario Basic Empathy Scale -BES (Jolliffe y Farrington, 2006), conformada por 8 ítems, organizados en un solo factor, que valora la dimensión cognitiva y afectiva de la empatía.

La prosocialidad se evaluó con la versión abreviada de 10 ítems, organizados unifactorialmente, pertenecientes a la Escala de prosocialidad-altruista (Roche, 1998). Esta escala valora aspectos como la ayuda física, servicio físico, dar y compartir, ayuda verbal, consuelo verbal, confirmación y valoración positiva del otro, escucha profunda, solidaridad, presencia positiva y unidad.

Para medir las metas sociales, se utilizó la escala Social Achievement Goal (Ryan y Shim, 2006) que fue utilizada en un estudio con una muestra colombiana (Romera et al., 2017). Dicha escala está conformada por 12 ítems que se organizan en tres factores (4 ítems para cada uno) así: metas sociales de aproximación, metas sociales de evitación y metas sociales de desarrollo/promoción.

Para valorar el ajuste normativo y el ajuste social se utilizaron 12 ítems organizados en dos factores (4 de ajuste normativo y 8 de ajuste social) que pertenecen al Cuestionario de 
Convivencia Escolar (Córdoba et al., 2016). El ajuste social se asumió como la calidad de las relaciones interpersonales entre iguales.

Finalmente, para medir la autopercepción de eficacia en el desempeño social se aplicó la escala de autoeficacia social perteneciente al Cuestionario Multidimensional de Competencia Social para Adolescentes -AMSC-Q (GómezOrtiz et al., 2017) y utilizado en un estudio con una muestra colombiana (Romera et al., 2017). Esta escala de 4 ítems valora la percepción de eficacia en las relaciones con los compañeros de clase, con los profesores, con familiares y con otros adultos. Los ítems de la escala fueron asumidos como variables categóricas observadas para ser incluidas en el modelo de análisis explicativo-predictivo.

Para todos los instrumentos se corroboraron sus propiedades psicométricas calculando su consistencia interna y realizando análisis factoriales exploratorios (en adelante AFE) y confirmatorios (en adelante AFC) para corroborar su estructura y poder utilizarlos en los análisis estadísticos. Los resultados se presentan más adelante en el apartado de valoración de las propiedades psicométricas y validación de las escalas. Todas las escalas fueron de tipo likert con valoración de "1" a "7", donde "1" indica que el comportamiento o enunciado es "totalmente falso", y "7" que es "totalmente verdadero".

\section{PROCEDIMIENTO}

La investigación tuvo un diseño transversal, ex post facto retrospectivo, un grupo, múltiples medidas (Montero y León, 2007). Tras obtener los permisos adecuados por parte de los equipos directivos, se visitaron los colegios para entregar y firmar el consentimiento informado por parte de los padres y el asentimiento por parte de los estudiantes. Una vez firmado estos formatos, se procedió a administrar el cuestionario. Se explicó a los alumnos el objetivo del estudio y se insistió al alumnado sobre el carácter anónimo, confidencial y voluntario de su participación. El tiempo medio de cumplimentación de la batería fue de 30 minutos. Todos los procedimientos se realizaron en conformidad con la declaración de Helsinki de 1964 y sus modificaciones posteriores o estándares éticos comparables; también con lo establecido en materia de investigación por la Ley 1090 del 2006 del ejercicio del psicólogo en Colombia y la Resolución número 8430 de 1993 por la cual se establecen las normas científicas, técnicas y administrativas para la investigación en salud.

\section{ANÁLISIS DE DATOS}

En primer lugar, se procedió a la validación de las escalas utilizadas para conocer su estructura factorial. Para ello, se dividió la muestra en dos partes, tomando el género como variable de selección, con un número proporcional de chicos y chicas (Neukrug y Fawcett, 2014). Esto permitió la realización de análisis factoriales exploratorios (AFE) y confirmatorios (AFC) con el procedimiento de validación cruzada, que permite optimizar la generalización del modelo a través del uso de dos sub-muestras escogidas aleatoriamente (Delgado-Rico et al., 2012; Lloret-Segura et al., 2014). Los criterios de valoración para el AFE fueron: el índice de adecuación muestral KMO- Kaiser-Meyer-Olkin (valores por encima de .6 son adecuados), el test de esfericidad de Bartlett (valor $p \leq .001$ ) y la varianza total explicada. Para el proceso de adecuación de ítems se consideró mantener los ítems que presentaran comunalidades mayores a .30, y un peso factorial inferior a .40 (Worthington y Whittaker, 2006); igualmente, los criterios aplicados en el AFC fue mantener aquellos ítems con cargas factoriales iguales 0 superiores a .30 y errores de medición que no excedan el .80 (Flora y Curran, 2004).

El AFE se llevó a cabo con el programa Factor 9.2 (Lorenzo-Seva y Ferrando, 2006) utilizando el método de estimación Unweighted Least Squares (ULS), el método de rotación oblicuo y 
tomando como base la matriz de correlaciones policóricas, puesto que todas las variables del cuestionario eran categóricas y reflejaron ausencia de normalidad multivariante (Bryant y Satorra, 2012). Este aspecto también determinó que el análisis de consistencia interna de cada una de las escalas se hiciera a través del Omega de McDonald ( $($ ) (Elosua-Oliden y Zumbo, 2008) calculado con el mismo programa (debe ser igual o superior a .6).

Para confirmar la estructura factorial sugerido por el AFE, se realizó un AFC con cada una de las escalas (Byrne, 2006) utilizando el método de estimación Maximum likelihood con la corrección robusta de Satorra-Bentler (Bentler, 2005). Para evaluar el ajuste del AFC se consideró el valor de significatividad del chi-cuadrado de Satorra-Bentler $\left({ }_{\mathrm{S}-\mathrm{B}}\right)$ y el chi-cuadrado normado o dividido por los grados de libertad (valores menores a 3 son adecuados). Como el valor de este índice está sujeto a ciertas variables como el tamaño de la muestra (Byrne, 2006), también se consideraron otros indicadores como el Compartive Fit Index (CFI), el Non-Normed Fit Index (NNFI) (valores superiores a .95 indican un buen ajuste) y el Root Mean Square Error Aproximation (RMSEA) (valores inferiores a .08 indican un buen ajuste) (Bentler, 2005; Byrne, 2006; Hu y Bentler, 1999). Finalmente, para dar respuesta al objetivo del estudio se realizó un Structural Equation Model - SEM (Kline, 2011). Los análisis en mención se realizaron con el programa estadístico EQS 6.2 (Bentler, 2005).

El nivel de significatividad adoptado para los CFA y el modelo SEM fue de .05 .

\section{VALORACIÓN DE LAS PROPIEDADES PSICOMÉTRICAS Y VALIDACIÓN DE LAS ESCALAS.}

La escala de empatía Basic Empathy Scale $B E S$ de Jolliffe y Farrington (2006) mostró una medida de adecuación muestral Kaiser-MeyerOlkin $K M O=.86$ y test de esfericidad de Bartlett significativo $(2 /(28)=599.845 ; p<.001)$. En el AFE se obtuvo un modelo unifactorial que explicó el $38.82 \%$ de la varianza. Con esta organización unifactorial se procedió a realizar el AFC obteniendo índices que demuestran un buen ajuste: ${ }_{\mathrm{S}-\mathrm{B}}^{2}=32.296 ;{ }_{\mathrm{S}-\mathrm{B}} \mathrm{B}(20)=1.615 ; p=.040$; $N N F I=.986 ; C F l=.990 ; R M S E A=.050(90 \% \mathrm{Cl}$ $[.043, .055])$. La consistencia interna calculada con el Omega de McDonald fue adecuada $\left(\omega_{\text {empatia }}=.86\right)$. Las propiedades psicométricas reconocidas son coherentes con los referentes teóricos y un adecuado ajuste al modelo propuesto por los autores. En consecuencia, se utilizó una escala conformada por 8 ítems tomados de la escala original organizados en un solo factor.

La escala de prosocialidad utilizada fue la escala de Prosocialidad-altruismo de Roche (1998). La extracción en el AFE arrojó un solo factor que explicó el $40.61 \%$ de la varianza. La escala validada obtuvo un $K M O=.86$ y test de esfericidad de Bartlett significativo $\left({ }^{2} /(45)=\right.$ 664.270; $p<.001)$. En coherencia con lo anterior en el AFC se obtuvieron adecuados índices de ajuste para el modelo unifactorial: ${ }^{2} \mathrm{~S}-\mathrm{B}=43.640$; ${ }_{\mathrm{S}-\mathrm{B}} /(35)=1.247 ; p=.145 ; \mathrm{NNFI}=.996 ; \mathrm{CFI}$ $.997 ;$ RMSEA $=.032(90 \% \mathrm{Cl}[.028, .039]) . \mathrm{La}$ consistencia interna también fue adecuada $\left(\oplus_{\text {prosocialidad }}=.84\right)$.

Los resultados del AFE de la escala de metas sociales Social Achievement Goal (Ryan y Shim, 2006) fueron aceptables, mostrando un $K M O=$ .80 y test de esfericidad de Bartlett significativo $\left({ }^{2} /(66)=1015.701 ; p<.001\right)$. En el análisis del AFE se obtuvieron tres factores (similar a la escala original) que explicaron el $48.34 \%$ de la varianza. Los índices de ajuste obtenidos en el AFC confirmaron la adecuación de dicha estructura factorial: ${ }_{\mathrm{S}-\mathrm{B}}=84.106 ;{ }_{\mathrm{S}-\mathrm{B}} \mathrm{B}(51)=1.649$; $p=.002 ; \mathrm{NNFl}=.975 ; \mathrm{CFl}=.980 ; \mathrm{RMSEA}=.051$ $(90 \% \mathrm{Cl}[.048, .057])$. La consistencia interna del instrumento fue adecuada $\left(\omega_{\text {metas-promoción }}=.80\right.$, $\left.\Phi_{\text {metas-aproximación }}=.90, \Phi_{\text {metas-evitación }}=.76\right)$. 
Para medir el ajuste normativo y el ajuste social se aplicó el Cuestionario de Convivencia Escolar (Córdoba et al., 2016) que incluye la medición de estas dos dimensiones. El AFE mostró un $K M O=$ .80 y test de esfericidad de Bartlett significativo $(2 /(66)=856.107 ; p<.001)$. En la extracción del AFE se obtuvieron dos factores (similar con el cuestionario original) que explicaron el $38.80 \%$ de la varianza. Los índices de ajuste del AFC fueron adecuados: ${ }^{2}{ }_{\mathrm{S}-\mathrm{B}}=97.648 ;{ }_{\mathrm{S}-\mathrm{B}} /(53)=1.842$; $p=.00018 ;$ NNFI $.977 ; \mathrm{CFl}=.981 ; \mathrm{RMSEA}=$ $.059(90 \% \mathrm{Cl}[.053, .055])$. La consistencia interna también fue adecuada $\left(\omega_{\text {ajuste-social }}=.85\right.$, $\Phi_{\text {ajuste-normativo }}=.83$ ).

Los resultados del AFE de la escala de autoeficacia social perteneciente al Cuestionario Multidimensional de Competencia Social para Adolescentes-AMSC-Q (Gómez-Ortiz et al., 2017) fueron aceptables, mostrando un $K M O=$
.83 y test de esfericidad de Bartlett significativo $\left({ }^{2} /(45)=968.932 ; p<.001\right)$. En el análisis del AFE se obtuvo una distribución unifactorial (similar a la escala original) que explicó el $45.82 \%$ de la varianza total. Los índices de ajuste obtenidos en el AFC confirmaron la adecuación de la estructura unifactorial: ${ }_{\mathrm{S}-\mathrm{B}}^{2}=148.375 ;{ }_{\mathrm{S}-\mathrm{B}}^{2} /(74)=$ 2.005; $p=.002 ; \mathrm{NNFI}=.980 ; \mathrm{CFI}=.984 ; \mathrm{RMSEA}$ $=.064(90 \% \mathrm{Cl}[.049, .079])$. La consistencia interna de la escala fue adecuada ( $\omega_{\text {autoeficacia- }}$ social $=.84)$.

\section{RESULTADOS}

Para ofrecer una visión general de los constructos analizados, se presentan las medias y desviaciones típicas (ver Tabla 1) de cada una de las escalas utilizadas que constituyeron las variables del modelo predictivo-explicativo.

\section{Tabla 1.}

Medias y desviaciones típicas de cada escala.

Escala

Metas sociales de aproximación

Metas sociales de promoción/desarrollo

Metas sociales de evitación

Ajuste social

Ajuste normativo

Prosocialidad

Empatía

Autoeficacia social

\begin{tabular}{ll}
\multicolumn{2}{c}{$\mathrm{n}(1084)$} \\
\hline$M$ & $D . T$. \\
3.60 & 1.35 \\
5.65 & .912 \\
4.53 & 1.439 \\
5.90 & .798 \\
5.77 & 1.134 \\
6.34 & 1.002 \\
5.25 & 1.171 \\
5.92 & 1.001 \\
\hline
\end{tabular}

Posteriormente, se obtuvo un modelo de ecuaciones estructurales -SEM (ver Figura 1) que permitió, además de establecer las influencias, reconocer los valores predictivos de las dimensiones para analizarlas de manera integral (Kline, 2011).

El modelo final mostró que las variables que influyeron directamente sobre el ajuste social fueron: la autopercepción de eficacia en el desempeño social con los compañeros $(=.37$; $p<.05)$, la prosocialidad $(=.25 ; p<.05)$ y la empatía $(=.13 ; p<.05)$. Estas tres variables se organizaron como variables mediadoras. De igual manera, influyeron directamente, la autopercepción de eficacia en el desempeño social con la familia $(=.15 ; p<.05)$, las metas de promoción $(=.14 ; p<.05)$, el ajuste normativo $(=.14 ; p<.05)$, la autopercepción de eficacia 
en el desempeño social con profesores (= $-.10 ; p<.05)$, las metas de aproximación (= $.19 ; p<.05)$ y la autopercepción de eficacia en el desempeño social con adultos $(=.10$; $p<$.05). De manera indirecta, influyeron en el ajuste social, a través de su relación con la prosocialidad, las metas de promoción $(=.38$; $p<.05)$, el ajuste normativo $(=.37 ; p<.05)$, la empatía ( $=.20 ; p<.05)$, la autopercepción de eficacia en el desempeño social con adultos $(=.13 ; p<.05)$, la autopercepción de eficacia en el desempeño social con profesores $(=.08$; $p<.05)$, y la autopercepción de eficacia en el desempeño social con la familia $(=-.02 ; p<.05)$. Estas relaciones permitieron explicar el $49 \%$ de la varianza de la prosocialidad.

Figura 1.

Modelo final SEM para explicar la calidad de las relaciones interpersonales entre iguales $\left({ }^{*} p<.05\right)$.

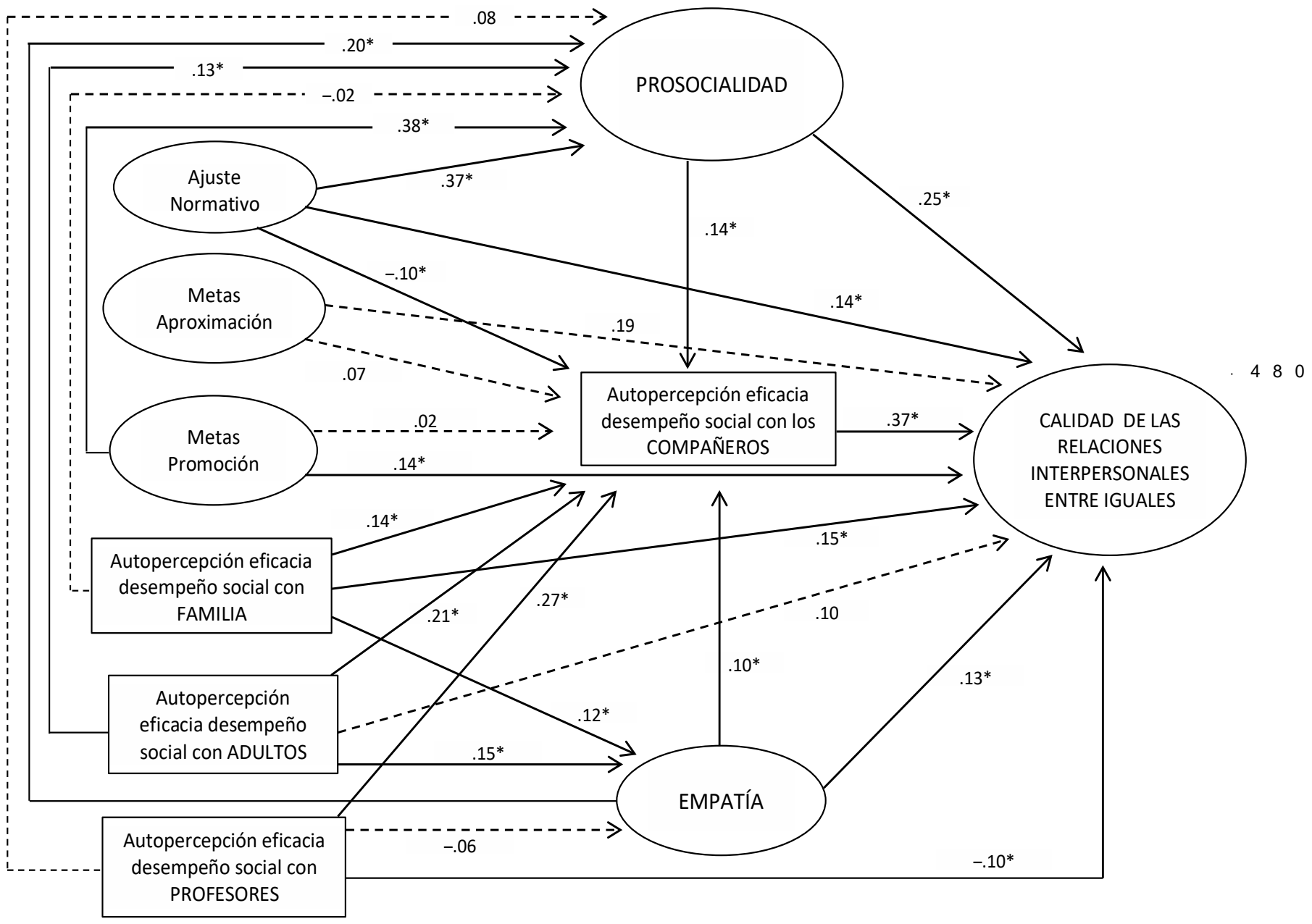


A través de su relación con la autopercepción de eficacia en el desempeño social con los compañeros, influyeron de manera indirecta en el ajuste social, la prosocialidad $(=.14 ; p<.05)$, la empatía $(=.10 ; p<.05)$, la autopercepción de eficacia en el desempeño social con los profesores $(=.27 ; p<.05)$, la autopercepción de eficacia en el desempeño social con los adultos $(=.21 ; p<.05)$, la autopercepción de eficacia en el desempeño social con la familia $(=.14 ; p<.05)$, el ajuste normativo $(=-.10 ; p<$ $.05)$, las metas de aproximación $(=.07 ; p<.05)$ y las metas de promoción $(=.02 ; p<.05)$. Todas estas relaciones permitieron explicar el $37 \%$ de la varianza de dicha variable.

Por su parte, a través de su relación con la empatía, influyeron de manera indirecta en el ajuste social, la autopercepción de eficacia en el desempeño social con los adultos $(=.15$; $p<.05)$, la autopercepción de eficacia en el desempeño social con la familia $(=.12 ; p<.05)$ y la autopercepción de eficacia en el desempeño social con los profesores $(=-.06 ; p<.05)$. Estas relaciones explicaron el $5 \%$ de la varianza de la empatía.

Todas estas relaciones directas e indirectas consiguieron explicar el $64 \%$ de la varianza de la calidad de las relaciones interpersonales entre iguales. Los índices obtenidos mostraron un buen ajuste del modelo: ${ }_{\mathrm{S}-\mathrm{B}}=1175.990 ;{ }_{\mathrm{S}-\mathrm{B}} /$ $(950)=1.238 ; p<.001 ; C F I=.961 ; N N F I=.958$; RMSEA $=.022(90 \% \mathrm{Cl}[.020, .027])$.

\section{DISCUSIÓN}

El objetivo del estudio fue determinar las relaciones e influencias, por tanto el valor predictivo y explicativo, de algunas dimensiones de la psicología del sujeto y del contexto, en la calidad de las relaciones interpersonales entre adolescentes escolarizados en la ciudad de Pasto, Colombia. En este sentido, los análisis mostraron que efectivamente la prosocialidad, la empatía, las metas de promoción y aproximación, el ajuste normativo y la autopercepción de eficacia en el desempeño social predicen, en conjunto, la calidad de dichas relaciones.

Los resultados indicaron que la calidad de las relaciones interpersonales entre iguales recibe influencia directa de la prosocialidad, la autopercepción de eficacia en el desempeño social con los compañeros y la empatía, dominios que además se configuraron como variables mediadoras. Esto coincide con los estudios que sugieren que actitudes como la solidaridad, el apoyo mutuo, el respeto (Buhrmester et al., 1988; Eisenberg et al., 2006), la influencia de los iguales (Day et al., 2014; Maras, 2007) y el entendimiento e implicación emocional influyen positivamente en el establecimiento de relaciones interpersonales de los adolescentes (Hay et al., 2021; Gutiérrez et al., 2011).

El análisis también indicó influencias directas por parte de la autopercepción de eficacia en el desempeño social con la familia, las metas sociales de promoción y el ajuste normativo, sobre el ajuste social. Esto sugiere que la autopercepción de eficacia en el desempeño social con los padres y otros familiares, cumplir y ajustarse a las normas, esforzarse por mejorar las relaciones interpersonales y buscar amigos que promuevan nuevos aprendizajes sociales, aportan positivamente al establecimiento de relaciones interpersonales de calidad. Esto es coherente con los estudios que destacan el papel de los padres y los iguales en los procesos de aprendizaje y desarrollo social (Miklikowska et al., 2011; Kingery et al., 2011), y los que sugieren que las regulaciones normativas influyen positivamente en el logro de relaciones interpersonales de calidad (Hamm et al., 2013; Moutousis et al., 2014). Sin embargo, es necesario subrayar que el modelo mostró una influencia directa negativa de la autopercepción de eficacia en el desempeño social con los profesores sobre el ajuste social, sugiriendo que quizás para los estudiantes, las buenas 
relaciones interpersonales con los profesores no siempre favorecen el establecimiento de relaciones interpersonales positivas con sus compañeros. Al respecto, algunos estudios mencionan que esto se debe a que el estudiante relaciona más al profesor con el rol de evaluación, supervisión y castigo, restando importancia al rol de conciliación (Day et al., 2014; Lessard et al., 2021).

El estudio mostró influencias indirectas sobre el ajuste social por parte de las autopercepciones de eficacia en el desempeño social con la familia, los adultos y los profesores, la prosocialidad y la empatía, a través de la autopercepción de eficacia en el desempeño social con los compañeros. Esto sugiere por un lado que, para el adolescente sentirse eficaz en las relaciones interpersonales con los padres, otros adultos y profesores asociado con actitudes prosociales y empáticas, predice la autopercepción de eficacia en el desempeño social con los compañeros; y por otro, que los aprendizajes sociales adquiridos trascienden, se replican y retroalimentan de un ámbito a otro (Caprara y Steca, 2005; van der Storm et al., 2021). Es necesario resaltar la influencia directa negativa del ajuste normativo sobre la autopercepción de eficacia en el desempeño social con los compañeros; esto sugiere que, desde la perspectiva del estudiante, ajustarse a las normas podría no siempre favorecer el sentirse eficaz en la interacción con sus iguales, debido quizás a que dichas normas se perciben restrictivas y limitantes (Cillessen y Rose, 2005). Esto, analizado en conjunto con el aporte directo observado hacia el ajuste social, sugiere dos vías de influencia de la normatividad: a) una que refiere un aporte positivo a la calidad de las relaciones, y b) otra negativa hacia la autopercepción de eficacia en el desempeño social con los iguales (Bowker y Spencer, 2010).

El análisis también indicó influencias indirectas sobreelajustesocial, através delas prosocialidad, por parte de las metas de promoción, el ajuste normativo, la empatía y la autopercepción de eficacia en el desempeño social con los adultos. Esto sugiere que los esfuerzos por desarrollar relaciones interpersonales positivas, buscar amigos que promuevan nuevos aprendizajes relacionales, la disposición a entender e implicarse en las emociones de los demás, y percibirse eficaz en las relaciones sociales con otros adultos predice de manera importante la conducta y actitud prosocial, mediando, a su vez, beneficios en el establecimiento de relaciones interpersonales entre iguales (Gilman y Anderman, 2006; Li y Wright, 2014; Rodkin y Ryan, 2012). Igualmente, se encontraron influencias indirectas de la autopercepción de eficacia en el desempeño social con la familia y otros adultos, a través de la empatía, sobre el ajuste social corroborando nuevamente la relevancia de ésta en el desarrollo social de los adolescentes (Dupont et al., 2014; Van-Lissa et al., 2014).

En conclusión, el estudio mostró que algunas dimensiones de la psicología del sujeto y otras del contexto predicen la calidad de las relaciones interpersonales entre iguales en la escuela, destacándose entre las estudiadas, la prosocialidad, la empatía y la autoeficacia social con los compañeros; estas variables deben ser consideradas de manera relevante en la implementación de programas para el mejoramiento de la convivencia y clima escolar, pues, a la luz de los resultados, predicen el mejoramiento de las relaciones interpersonales entre iguales.

Otro valor agregado del estudio refiere que las escalas de empatía, prosocialidad, metas sociales, autoeficacia social y ajustes social y normativo, utilizadas en el estudio, mostraron óptimas propiedades psicométricas, además de adecuados valores de consistencia 
interna. Esto ofrece al contexto colombiano escalas homologadas internacionalmente y de reconocida calidad psicométrica.

Se reconocen algunas limitaciones referidas a la transversalidad del estudio, el uso y aplicación de auto-registros y el método de validación de las escalas. El estudio sugiere la necesidad de explorar en mayor profundidad la influencia de los profesores en el establecimiento de las relaciones interpersonales asociadas a la amistad y al compañerismo.

\section{REFERENCIAS BIBLIOGRÁFICAS}

Bandura, A. (2001). Social Cognitive Theory: An Agentic Perspective. Annual Review of Psychology, 52, 1- 26. doi.org/10.1146/ annurev.psych.52.1.1

Bandura, A., Caprara, G.V., Barbaranelli, C., Gerbino, M., \& Pastorelli, C. (2003). Role of Affective Self-Regulatory Efficacy in Diverse Spheres of Psychosocial Functioning. Child Development, 74(3), 769-782. doi.org/10.1111/14678624.00567

Bentler, P. M. (2005). EQS structural equations program manual. Encino, CA: Multivariate Software.

Bowker, J. C., \& Spencer, S. V. (2010). Friendship and Adjustment: A Focus on Mixed-Grade Friendships. Journal of Youth and Adolescence, 39, 1318-1329. doi: 10.1007/s10964-009-9474-0

Bronfenbrenner, U. (2005). Making human beings human. Bioecological perspectives on human development. Thousand Oaks, CA: Sage.
Brouzos, A., Misailidi, P., \& Hadjimattheou, A., (2014). Associations Between Emotional Intelligence, SocioEmotional Adjustment, and Academic Achievement in Childhood: The Influence of Age. Canadian Journal of School Psychology, 29(2), 83-99. doi. org/10.1177/0829573514521976

Bryant, F. B., \& Satorra, A. (2012). Principles and practice of scaled difference Chi-Square testing. Structural Equation Modeling: $A$ Multidisciplinary Journal, 19(3), 372-398. DOI: 10.1080/10705511.2012.687671

Buhrmester, D., Furman, W., Wittenberg, M., \& Reis, H. (1988). Five Domains of Interpersonal Competence in Peer Relationships. Journal of Personality and Social Psychology, 55(6), 991-1008.

Byrne, B. M. (2006). Structural Equation Modeling with EQS. Basic concepts, applications, and programming. New York - London: 2nd ed. Psychology Press.

Caprara, G.V., \& Steca, P. (2005). Effective and social self-regulatory efficacy beliefs as determinants of positive thinking and happiness. European Psychologist, 10(4), 275-286. DOI: 10.1027/10169040.10 .4 .275

Carré, A., Stefaniak, N., D'Ambrisio, F., Bensalah, L., \& Besche-Richard, C. (2013). The Basic Empathy Scale in Adults (BES-A): Factor Structure of a Revised Form. Psychological Assessment, American Psychological Association, 25(3), 679691. doi:10.1037/a0032297

Casas, J. A., Ruiz-Olivares, R., \& Ortega-Ruiz, R. (2013). Validation of the Internet and Social Networking Experiences. Questionnaire in Spanish adolescents. International Journal of Clinical and Health Psychology, 13(1), 40-48. doi. org/10.1016/S1697-2600(13)70006-1 
Casas, J., Del Rey, R., \& Ortega-Ruiz, R. (2013). Bullying and cyberbullying: Convergent and divergent predictor variables. Computers in Human Behavior, 29(3), 580-587. doi.org/10.1016/j. chb.2012.11.015

Cillessen, A. H. N., \& Rose, A. J. (2005). Understanding popularity in the peer system. Current Directions in Psychological Science, 14(2), 102-105. doi.org/10.1111/j.09637214.2005.00343.x

Connolly, J. (1989). Social self-efficacy in adolescence: Relations with self concept, social adjustment, and mental health. Canadian Journal of Behavioral Science, 21(3), 258-269. http://dx.doi.org/10.1037/ h0079809

Córdoba, A., Del Rey, R., Casas, J., \& Ortega, R. (2016). Valoración del alumnado de primaria sobre convivencia escolar: El valor de la red de iguales. Psicoperspectivas, 15(2), 78-89. Doi:10.5027/psicoperspectivas-Vol15Issue2-fulltext-760

Day, K., Hamm, J., Lambert, K., \& Farmer, T. (2014). Social Integration and Students' Perceptions of the Transition to Middle School. Journal of Educational and Developmental Psychology, 4(1), 10-26. doi:10.5539/jedp.v4n1p10

Decety, J., \& Svetlova, M. (2012). Putting together phylogenetic and ontogenetic perspectives on empathy. Developmental Cognitive Neuroscience, 2(1), 1-24. doi: 10.1016/j.dcn.2011.05.003

Delgado-Rico, E., Carretero-Dios., H., \& Ruch, W. (2012). Content validity evidences in test development: An applied perspective. International Journal of Clinical and Health Psychology, 12(3), 449-460.
Del Rey, R., Ortega, R., \& Feria I., (2008). Riesgos de la violencia escolar. Revista Informe de Investigaciones Educativas, 22(2), 257-273.

Dupont S., Galand B., Nils F., \& Hospel V. (2014). Social Context, Self-perceptions and Student Engagement: A SEM investigation of the selfsystem model of motivational development (SSMMD). Electronic Journal of Research in Educational Psychology, 12(1), 5-32

Eisenberg, N., Fabes, R. A., \& Spinrad, T. L. (2006). Prosocial development. In W. Damon y N. Eisenberg (Eds.), Handbook of Child Psychology: Social, Emotional and Personality Development (Vol. 3, pp. 646-718). Nueva York: John Wiley y Sons.

Elosua-Oliden P., \& Zumbo, B. D. (2008). Coeficientes de fiabilidad para escalas de respuesta categórica ordenada. Psicothema, 20(4), 896-901.

Elledge, L. C., Williford, A., Boulton, A., J., DePaolis, K., J., Little, T., D., \& Salmivalli, C. (2013). Individual and Contextual Predictors of Cyberbullying: The Influence of Children's Provictim Attitudes and Teachers' Ability to Intervene. Journal of Youth and Adolescence, 42(5), 698-710. DOI: $10.1007 / \mathrm{s} 10964-013-9920-x$

Fernández-Pinto I., López-Pérez B., \& Márquez M., (2008). Empatía: Medidas, teorías y aplicaciones en revisión. Anales de Psicología, 24(2), 284-298.

Flora, D. V., \& Curran, P. J. (2004). An Empirical Evaluation of Alternative Methods of Estimation for Confirmatory Factor Analysis With Ordinal Data. Psychological Methods, 9(4), 466-491. doi:10.1037/1082-989X.9.4.466 
García, F. J., Sureda, I., \& Monjas, Mª I. (2010). El rechazo entre iguales en la Educación Primaria: Una panorámica general. Anales de Psicología, 26(1), 123-136.

Gilman, R., \& Anderman, E. M. (2006). The relationship between relative levels of motivation and intrapersonal, interpersonal, and academic functioning among older adolescents. Journal of School Psychology, 44(5), 375-391. https://doi.org/10.1016/j.jsp.2006.03.004

Giddens A. (1984). The Constitution of Society: Outline of the Theory of Structuration. Cambridge: Polity/Berkeley: Univ. Calif. Press.

Gómez-Ortiz, O., Romera, E., \& Ortega-Ruiz, R. (2017). Multidimensionalidad de la competencia social: Medición del constructo y su relación con los roles del bullying. Revista de Psicodidáctica, 22(1). 37-44. doi.org/10.1016/S11361034(17)30042-4

Gutiérrez, M., Escartí A., \& Pascual C. (2011). Relaciones entre empatía, conducta prosocial, agresividad, autoeficacia $y$ responsabilidad personal y social de los escolares. Psicothema, 23(1), 13-19.

Hamm, J. V., \& Faircloth, B. S. (2005). The role of friendship in adolescents' sense of school belonging. New Directions for Child and Adolescent Development, 107, 61-78. DOI:10.1002/cd.121

Hamm, J. V., Lambert, K., Agger, C. A., \& Farmer, T. W. (2013). Promotive contexts of academic and social adjustment among rural African American early adolescent boys. Journal of Orthopsychiatry, 83, 278288. doi:10.1111/ajop.12030.

Hartup, W. W. (2005). Peer interaction: What causes what?. Journal of Abnormal Child Psychology, 33(3), 387-394.
Hay, D. F., Paine, A. L., Perra, O., Cook, K. V., Hashmi, S., Robinson, C., ... \& Slade, R. (2021). Prosocial and aggressive behavior: a longitudinal study. Monographs of the society for research in child development, 86(2), 7-103.

Herrera, M., Romera, E., Ortega, R., \& Gómez, O. (2016). Influence of Social Motivation, Self-perception of Social Efficacy and Normative Adjustment in the peer setting. Psicothema, 28(1), 32-39. doi:10.7334/ psicothema2015.135

Hinduja, S., \& Patchin, J., W. (2013). Social Influences on Cyberbullying Behaviors Among Middle and High School Students. Journal of Youth and Adolescence, 42(5),711-722. doi:10.1007/s10964-012$9902-4$

Hu, L. T., \& Bentler, P. M. (1999). Cutoff criteria for fit indexes in covariance structure analysis: Conventional criteria versus new alternatives. Structural Equation Modeling: A Multidisciplinary Journal, 6(1), 1-55. https://doi. org/10.1080/10705519909540118

Jolliffe, D., \& Farrington, D. P. (2006). Development and validation of the Basic Empathy Scale. Journal of Adolescence, 29(4), 589-611. https://doi.org/10.1016/j. adolescence.2005.08.010

Kingery, J. N., Erdley, C. A., \& Marshall, K. C. (2011). Peer acceptance and friendship as predictors of early adolescents' adjustment across the middle school transition. Merrill-Palmer Quarterly, 57(3), 215-243. DOI:10.1353/mpq.2011.0012

Kline, R. (2011). Principles and practice of Structural Equation Modeling. New York: Guildford Press. 
La Greca, A. M., \& López, N. (1998). Social anxiety among adolescents: Linkages with peer relations and friendships. Journal of Abnormal Child Psychology, 26(2), 83-94.

Lessard, L. M., Lawrence, S. E., \& Puhl, R. M. (2021). Weight-based victimization and school performance in adolescence: Can teachers help reduce academic risks?. School Psychology, 36(1), 69.

Li, Y., \& Wright, M. F. (2014). Adolescents' Social Status Goals: Relationships to Social Status Insecurity, Aggression, and Prosocial Behavior. Journal of Youth and Adolescence, 43(1), 146-160. doi:10.1007/s10964-013-9939-z

Lloret-Segura, S., Ferreres-Traver, A., Hernández-Baesa, A., \& Tomás-Marco, I. (2014). El análisis factorial exploratorio de los ítems: una guía práctica, revisada y actualizada. Anales de Psicología, 30(3), 1151-1169. http://dx.doi.org/10.6018/ analesps.30.3.199361

Lorenzo-Seva, U., \& Ferrando, P. J. (2006). FACTOR: A computer program to fit the exploratory factor analysis model. Behavioral Research Methods, Instruments and Computers, 38(1), 88-91. https://doi.org/10.3758/BF03192753

Maras, P. (2007). 'But no one in my family has been to University'Aiming Higher: School Students' Attitudes to Higher Education. The Australian Educational Researcher, 34(3), 69-90. https://doi.org/10.1007/ BF03216866

Martínez, Á. M., Jurado, M. D. M. M., del Carmen Pérez-Fuentes, M., Márquez, M. D. M. S., Martín, A. B. B., \& Linares, J. J. G. (2021). The complex nature of school violence: attitudes toward aggression, empathy and involvement profiles in violence. Psychology research and behavior management, 14, 575.
McGloin, J. M., Sullivan, C. J., \& Thomas, K. J. (2014). Peer Influence and Context: The Interdependence of Friendship Groups, Schoolmates and Network Density in Predicting Substance Use. Journal of Youth and Adolescence, 43(9), 14361452. doi:10.1007/s10964-014-0126-7

Méndez-Giménez, A., Cecchini, J., \& González C. (2012). Autodeterminación y metas sociales: un modelo estructural para comprender la intención de práctica, el esfuerzo y el aburrimiento en Educación Física. Aula Abierta, 40(1), 51-62.

Miklikowska, M., Duriez, B., \& Soenens, B. (2011). Family roots of empathy-related characteristics: The role of perceived maternal and paternal need support in adolescence. Developmental Psychology, 47(5), 1342-1352. doi:10.1037/a0024726

Montero, I., \& León, O. G. (2007). Guía para nombrar los estudios de investigación en Psicología. International Journal of Clinical and Health Psychology, 7(3), 847862.

Moutousis, M., Fearon, P., El-Deredy, W., Dolan, R., \& Friston, K., (2014). Bayesian inferences about the self (and others): $A$ review. Consciousness and Cognition, 25, 67-76. doi:10.1016/j.concog.2014.01.009

Neukrug, E., \& Fawcwtt, R. (2014). Essential of testing and assessment: A practical guide for counselors, social workers and psychologists. Stanford: Cengage Learning.

Ortega, R., \& Mora-Merchan, J. (1996). El aula como escenario de la vida afectiva y moral. Cultura y Educación, 8(3), 5-18. https:// doi.org/10.1174/113564096763270280 
Ortega, R., \& Mora-Merchan, J. (2008). Las redes de iguales y el fenómeno del acoso escolar: explorando el esquema dominio-sumisión. Infancia $y$ Aprendizaje, 31(4), 515-528. http://dx.doi. org/10.1174/021037008786140922

Patrick, H., Hicks, L., \& Ryan, A. M. (1997). Relations of perceived social efficacy and social goal pursuit to self-efficacy for academic work. Journal of Early Adolescence, 17(2), 109-128. https://doi. org/10.1177/0272431697017002001

Piaget, J. (1936). The origins of intelligence in children. New York: International Universities Press.

Poorthuis, A. M. G., Thomaes, S., Denissen, J. J. A., van Aken, M. A. G., \& Orobio de Castro, B. (2013). Personality in Action: Can Brief Behavioral Personality Tests Predict Children's Academic and Social Adjustment Across the Transition to Secondary School?. European Journal of Psychological Assessment, 30(3), 169177. http://dx.doi.org/10.1027/1015-5759/ a000186

Roche, R. (1995). Psicología y Educación para la Prosocialidad. Buenos Aires: Ciudad Nueva.

Roche, R. (1998). Educación Prosocial de las Emociones, Valores y Actitudes Positivas. Barcelona: Blume.

Rodkin, P. C., \& Ryan, A. M. (2012). Child and adolescent peer relations in educational context. In K. R. Harris, S. Graham, y T. Urdan (Eds.), APA education psychology handbook: Individual differences and cultural and contextual factors (Vol. 2, pp. 363-389). Washington, DC: American Psychological Association.
Rodkin, P., Ryan, A., Jamison R., \& Wilson T., (2013). Social Goals, Social Behavior, and Social Status in Middle Childhood. Developmental Psychology, 49(6), 11391150. doi:10.1037/a0029389

Romera, E., Herrera-López, M., Casas, J.A., Ortega-Ruiz, R., \& Gómez-Ortiz, O. (2017). Multidimensional Social Competence, Motivation, and Cyberbullying: A Cultural Approach with Colombian and Spanish Adolescents. Journal of Cross-Cultural Psychology, 48(8), 1183-1197. doi. org/10.1177/0022022116687854

Ryan, A. M., \& Shin, S., S. (2006). Social Achievement Goals: The Nature and Consequences of Different Orientations Toward Social Competence. Personality and SocialPsychologyBulletin, 32(9), 12461263. DOI:10.1177/0146167206289345

Segrin, C., \& Taylor, M. (2007). Positive interpersonal relationships mediate the association between social skills and psychological well-being. Personality and Individual Differences, 43(4), 637-646. https://doi.org/10.1016/j.paid.2007.01.017

Smithyman, T., Fyreman, G., \& Asher Y. (2014). Long-Term Psychosocial Consequences of Peer Victimization: From Elementary to High School. School Psychology Quarterly. American Psychological Association, 29(1), 64-76. doi:10.1037/ spq0000053.

van der Storm, L., van Lissa, C. J., Lucassen, N., Helmerhorst, K. O., \& Keizer, R. (2021). Maternal and Paternal Parenting and Child Prosocial Behavior: A Meta-Analysis Using a Structural Equation Modeling Design. Marriage \& Family Review, 1-37. 
Van-Lissa, C., Hawk, S., De Wied M., Koot, H., Van Lier, P., \& Meeus W., (2014). The Longitudinal Interplay of Affective and Cognitive Empathy Within and Between Adolescents and Mothers. Developmental Psychology, 50(4), 12191225. doi: $10.1037 / \mathrm{a} 0035050$

Worthington, R. L., \& Whittaker, T. A. (2006). Scale Development Research. A Content Analysis and Recommendations for Best Practices. The Counseling Psychologist, 34(6), 806-838. https://doi. org/10.1177/0011000006288127 